\title{
Graded Microstructure of Additive Manufactured Ti-6Al-4V via Electron Beam Melting
}

Hansheng Chen ${ }^{1,2,3}$, Christopher Cooper ${ }^{1}$, Cameron Durrant ${ }^{1}$, Yingluo Li $^{1}$, Sophie Primig ${ }^{4}$, Xiaozhou Liao $^{1}$, Sudarsanam Suresh Babu ${ }^{5,6}$ and Simon P. Ringer ${ }^{1,3^{*}}$

1. School of Aerospace, Mechanical, and Mechatronic Engineering, The University of Sydney, Sydney, NSW, Australia.

2. The University of Sydney Nano Institute, The University of Sydney, Sydney, NSW, Australia.

3. Australian Center for Microscopy and Microanalysis, The University of Sydney, NSW, Australia.

4. School of Materials Science and Engineering, The University of Sydney, Sydney, NSW, Australia.

5. Department of Mechanical, Aerospace, and Biomedical Engineering, University of Tennessee, Knoxville, Tennessee, TN, USA.

6. Manufacturing Demonstration Facility, Oak Ridge National Laboratory, Knoxville, TN, USA.

* Corresponding author: simon.ringer@sydney.edu.au

Electron beam melting (EBM) process is an additive manufacturing (AM) technique, which utilizes an electron beam to melt conductive metal powders under a high vacuum [1]. Typically, a raster/linear scan strategy is utilized in which the beam horizontally moves from left to right direction on the powder bed. Recently, a dehoff fill strategy which melts each $11^{\text {th }}$ point in a line, skips 5 lines, and repeats until each point is melted, was utilized to site-specifically control the crystallographic grain orientation in nickel base superalloy Inconel 718 systems [2]. However, whether the aforementioned phenomenon is universal in other alloy systems is still open to question. Here, we selected Ti-6Al-4V (one of the most vital engineering materials and has been applied in aerosapce and biomedical industry due to its high strengthto-weight ratio and ourstanding biocampatility) as the model system [3-5].

Ex-situ characterization experiments by light optical microscopy (LOM), scanning electron microscopy/electron backscatter diffraction/energy dispersive spectroscopy (SEM/EBSD/EDS), and atom probe tomography (APT) were performed on the dehoff EBM Ti-6Al-4V alloys. The LOM was utilized to reveal the morphology and microstructural evolution from top to bottom layers. The data collected using SEM/EBSD/EDS can be plotted in the form of maps, showing the grain size distribution, phase distribution, grain boundary information, crystallographic orientation, texture, and corresponding elemental distribution of top, middle and bottom layers. Figure 1 shows the inverse pole figure (IPF)-Y maps of $\alpha$-HCP phases in the top, middle, and bottom section of dehoff EBM Ti-6Al-4V alloys. an acicular $\alpha$ (HCP structure with $a=0.293 \mathrm{~nm}$ and $c=0.467 \mathrm{~nm})+$ rod-like $\beta$ (BCC structure with $a=0.320 \mathrm{~nm})$ lamellar microstructure was observed inside the prior $\beta$ grains. Five types of grain boundaries (GBs) were observed in the acicular $a$ phases in the top, middlde, and bottom layers, which are $<11-20>60^{\circ},<44$ $83>63.9^{\circ},<11-20>89.5^{\circ},<0001>10.2^{\circ},<11-20>29.8^{\circ}$. The proportion of $<11-20>60^{\circ} \mathrm{GBs}$ increases from $36 \%$ in the top layer to $42 \%$ in the bottom layer, while the proportion of $<44-83>63.9^{\circ} \mathrm{GBs}$ decreases from $25 \%$ in the top layer to $19 \%$ in the bottom layer. APT can detect individual atoms in three dimensions at the atomic scale and can be used to visualize the phase interfacial elemental variation at the nanoscale. The correlation between the microstructural evolution and elemental distribution of AM Ti-64 alloys with thermal gradients and gyrations will be discussed in detail [6]. 
References:

[1] WJ Sames et al., International Materials Reviews 61(5) (2016), p. 315.

[2] RR Dehoff et al., Materials Science and Technology 31(8) (2015), p. 931.

[3] W Xu et al., Acta Materialia 125 (2017), p. 390.

[4] R Sabban et al., Acta Materialia 162 (2019), p. 239.

[5] J Haubrich et al., Acta Materialia (2019).

[6] The authors acknowledge the facilities and the scientific and technical assistance of Microscopy Australia at the Australian Centre for Microscopy \& Microanalysis at the University of Sydney. The research was sponsored by the Department of the Navy, Office of Naval Research under ONR award number N00014-18-1-2794. Any opinions, findings, and conclusions or recommendations expressed in this material are those of the author(s) and do not necessarily reflect the views of the Office of Naval Research. The research was also sponsored by the US Department of Energy, Office of Energy Efficiency and Renewable Energy, Advanced Manufacturing Office under contract DE-AC0500OR22725 with UT-Battelle, LLC. Access to the Oak Ridge National Laboratory's (ORNL) additive manufacturing equipment at ORNL's Manufacturing Demonstration Facility (MDF) was facilitated by US Department of Energy's Strategic Partnership Projects (SPP) mechanism. More information can be found at https://science.energy.gov/lp/strategic-partnership-projects. Research sponsored by the U.S. Department of Energy, Office of Energy Efficiency and Renewable Energy, Industrial Technologies Program, under contract DE-AC05-00OR22725 with UT-Battelle, LLC.
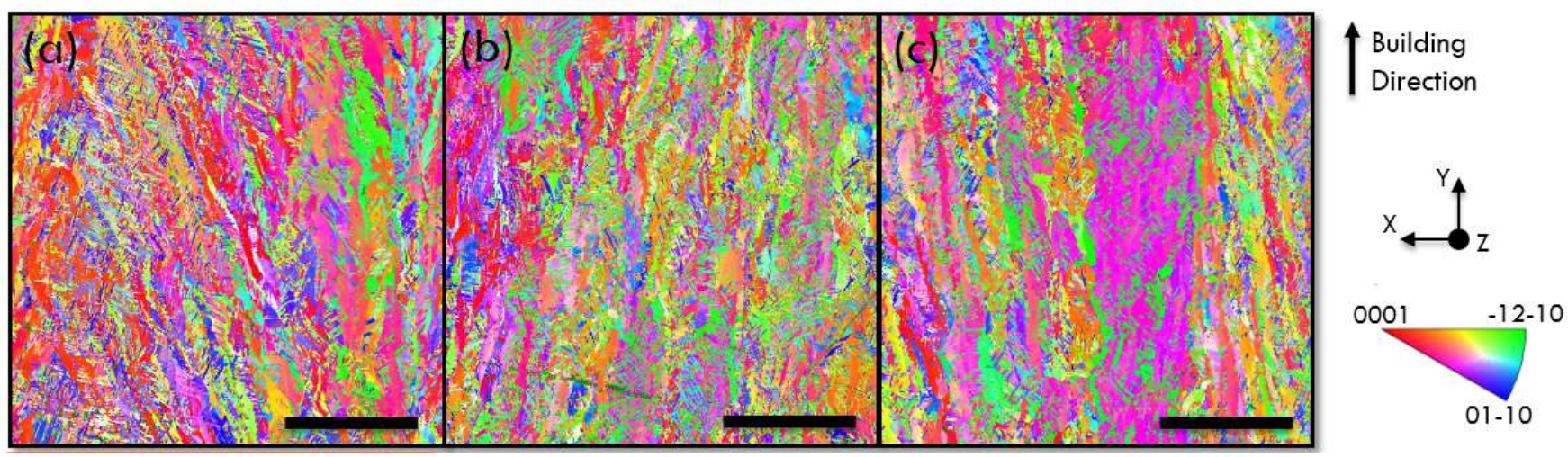

Figure 1. Microstructural evolution of dehoff EBM samples. Inverse pole figure (IPF)-Y maps of $\alpha$-HCP phase in the (a) top section, (b) middle section, and (c) bottom section. The color of each grain represents its orientation. The color cod is shown in the standard triangle (inset). The scale bar is $200 \mu \mathrm{m}$. 